\title{
Subjetividad, cotidianidad y memoria: la propuesta de documental colaborativo de José Balado*
}

\author{
Subjectivity, Quotidianness and Memory: The Proposal of a Collaborative Documentary by José Balado \\ Subjetividade, cotidianidade e memória: a proposta de um documentário colaborativo de José Balado
}

\author{
Salvador Leetoy ${ }^{\mathrm{a}}$ \\ Instituto Tecnológico y de Estudios Superiores de Monterrey, \\ México \\ sleetoy@tec.mx \\ ORCID: http://orcid.org/0000-0003-1203-0939 \\ Diego Zavala Scherer \\ Instituto Tecnológico y de Estudios Superiores de Monterrey, \\ México \\ ORCID: http://orcid.org/0000-0002-7362-4709
}

DOI: https://doi.org/10.11144/ Javeriana.syp 38-74.scmp Redalyc: http://www.redalyc.org/articulo.oa? $\mathrm{id}=86059657008$

Fecha de recepción: 17 Julio 2018 Fecha de aprobación: 30 Enero 2019 Fecha de publicación: 18 Junio 2019

\section{Resumen:}

Este texto analiza el caso de DOCUPERU, una organización de creación de contenidos audiovisuales, con el fin de reflexionar sobre el uso del documental colaborativo para la promoción de una cultura incluyente y de ciudadanía activa. Se establece que los productos diseñados por José Balado y su equipo son resultado de acciones colectivas que permiten a comunidades marginales acceder a formas de autorrepresentación, re-construir su memoria y hablar de su cotidianidad. Primero, se presenta un marco general sobre agencia cultural; después, se explica cómo estas formas de producción de amateurs han dado lugar a un nuevo impulso del documental colaborativo. Por último, se transcribe una entrevista con el propio Balado, donde explica los fundamentos que permiten crear esta vivencia colectiva.

Palabras clave: documental colaborativo, DOCUPERU, ciudadanía, agencia, autorrepresentación.

\section{Abstract:}

This paper analyzes the case of DOCUPERU, an organization that creates audiovisual contents intended to reflect on the use of the collaborative documentary, which aims to promote an inclusive culture of active citizens. It is determined that the products designed by José Balado and his team result from collective actions that allow the marginal communities to gain access to some ways of self-representation, rebuilding their memory and speaking of their quotidianness. Firstly this paper presents an overview of the cultural agency. Then, it explains how these forms of amateur production have led to a new boost for the collaborative documentary. Finally, an interview to Balado is transcribed herein; this way he explains the fundamentals making possible to create such collective life experience.

Keywords: collaborative documentary, DOCUPERU, citizenship, agency, self-representation.

\section{Resumo:}

Este texto analisa o caso de DOCUPERU, uma organização de criação de conteúdos audiovisuais, a fim de refletir sobre o uso do documentário colaborativo para a promoção de uma cultura inclusiva e de cidadania ativa. Estabelece-se que os produtos desenhados por José Balado e sua equipe são resultado de ações coletivas que permitem às comunidades marginais acessar a formas de auto representação, reconstruir sua memoria e falar da sua cotidianidade. Primeiro, apresenta-se um quadro geral sobre agencia cultural; depois explica-se como essas formas de produção de amateurs deram lugar a um novo impulso do documentário colaborativo. Por fim, transcreve-se entrevista com o próprio Balado, onde explica os fundamentos que permitem criar esta vivência coletiva.

Palavras-chave: documentário colaborativo, DOCUPERU, cidadania, agência, autorrepresentação.

Notas de autor:

a Autor de correspondencia. Correo electrónico: sleetoy@tec.mx 


\section{Introducción}

\section{Deliberación, participación, agencia}

Uno de los aspectos más atractivos de la teoría de la democracia deliberativa es, sin duda, que las decisiones de los ciudadanos sean sustentadas en el intercambio argumentativo que los empodera de frente a su propia condición social y política: alguien cuya voz es reconocida como interlocutor válido y valioso ante las instituciones, su comunidad y demás actores del entorno. Esto, asimismo, conduce a reconocer el papel pedagógico del diálogo como condición esencial para el aprendizaje social y político de ciudadanos, quienes a su vez aportan todo el saber acumulado de sus experiencias cotidianas (Held, 2007, pp. 333-335). Esta teoría, de carácter normativo, lo que sugiere son maneras de mejorar la democracia y criticar instituciones que no cumplen con dicha normativa: la democracia deliberativa es más justa y equitativa para lidiar con la diversidad y la pluralidad comparativamente con otros modelos de democracia, pues se desmarca de posiciones netamente individualistas e instrumentales, para dar paso al involucramiento y participación activa de ciudadanos en la determinación de la política pública. En términos concretos, la fortaleza de este enfoque es su sentido charlo-céntrico que privilegia la generación de discusiones y argumentaciones previas a cualquier toma de decisión (Chambers, 2003, p. 308).

Existe una vasta literatura que aborda la manera como estas prácticas comunicativas son elementos esenciales para la construcción de una democracia más incluyente y radical, la cual amplía sus alcances para analizar problemáticas diversas que afectan la participación ciudadana, ya sea para abrir canales de acción o para retar los impedimentos al reconocimiento pleno de sujetos agraviados por el sistema político (Benhabib, 2002; Dryzek, 2009; Elster, 2001; Fung, 2005; Velasco, 2006). Gran parte de la elaboración teórica sobre el tema ha surgido a propósito del pensamiento de Jürgen Habermas (1989), particularmente, sobre su estudio de la esfera pública, aquel espacio comunicativo en el cual los ciudadanos pueden lograr un diálogo racional sobre la vida social y los asuntos públicos, donde la información es fundamental para que sus participantes puedan intercambiar puntos de vista y se influyan mutuamente. No obstante, como reconoce el filósofo, lógicas instrumentales como aquellas propias del Estado y el mercado, basadas en la búsqueda o conservación del poder y el dinero, limitan o manipulan la cantidad y calidad de la información, lo que va en detrimento de la pluralidad en la conformación de opinión pública. Es decir, la posibilidad de construir formas de agencia ciudadana se ven acotadas por racionalidades técnicas sustentadas en relaciones de poder, por lo que es necesario una lógica distinta como fuerza emancipadora: una acción comunicativa originada a partir del intercambio dialéctico de quienes participan (Habermas, 1987). Así, dicha acción comunicativa se convierte en un concepto fundamental para determinar formas de resistencia a través de la exigencia por espacios de expresión e informativos que fomenten racionalidades contrarias a la dominación y la alienación.

De acuerdo a lo anterior, Nancy Fraser (1992) reformula la teoría de Habermas para, precisamente, discutir algo que el filósofo parece no elaborar del todo, y que resulta de la mayor importancia: la atención de esferas alternas marginadas al estar conformadas por grupos sociales no privilegiados, tal como pueden ser aquellas formas de exclusión determinadas por identidades de género, raza, grupo étnico, clase socioeconómica o preferencia sexual. A estas, Fraser las denomina contrapúblicos subalternos (p. 123), para enfatizar que son miembros de grupos sociales subordinados que crean y circulan contradiscursos, para formular interpretaciones oposicionales de sus identidades, intereses y necesidades. Por tanto, sin la voz del subalterno, cualquier intento de crear espacios democráticos deliberativos estará incompleto y será fallido. Ese es precisamente el punto que se quiere mostrar con el estudio del caso que aquí nos ocupa: ¿cómo crear puentes teóricos entre las ciencias sociales y las humanidades que diriman, por un lado, una problemática de falta de representatividad y, por el otro, la creación de formas de agencia que reten dicha carencia? La propuesta que aquí se presenta, basada en el caso de Documental Peruano ([DOCUPERU]; https://www 
.docuperu.pe/, es que ello se logra a través de la aspiración de una esfera pública incluyente, que alimente formas democráticas de deliberación, lograda a través de prácticas artísticas participativas. Como sostiene Doris Sommer (2014, p. 18, sin el placer que produce el arte, las reformas políticas y el pragmatismo político se marchitan en pretensiones contraproducentes y cortoplacistas.

La deliberación en la esfera pública, que luego conecte con espacios institucionales, no es del todo tersa y fluida. Concretamente, DOCUPERU ha intentado, a través de la producción y narrativa documental, visibilizar problemáticas que atañen a sujetos no privilegiados. Con sus acciones e intervenciones en comunidades marginadas, se constituyen como acción colectiva que aporta elementos de comprensión del impacto de políticas opresivas por parte del Estado y el mercado, ejerciendo formas de acción comunicativa que no se tejen de manera unilateral, sino, por el contrario, suceden de manera colaborativa en donde se fusiona el papel del creador de contenidos con el de actores que cuentan sus historias. Al respecto, y como bien lo han estudiado teóricos de los movimientos sociales (Boggs, 1986; Melucci, 1996; Offe, 1984), las acciones colectivas se dirigen a esferas de la vida social que no están solamente enfocadas a derechos cívicos o económicos, sino que, frecuentemente, incluyen derechos sociales tales como el respeto a la diferencia cultural y a la autodeterminación, el pluralismo y la no discriminación, el respeto a los derechos humanos, etcétera. Por ello, las historias contadas y compartidas sirven para ampliar el espectro deliberativo de la democracia, al incluir voces residuales que no logran tener acceso a otros canales de comunicación. No habría que olvidar que acciones por la diversidad sexual, contra la violencia hacia las mujeres, de determinación y soberanía indígena, de sustentabilidad y protección al ambiente, por una educación digna, etcétera, proveen de experiencia considerable para la confección de una política pública más digna, que se canaliza a través de diversas formas de agencia cultural (Sommer, 2006, 2014).

Si la agencia humana puede ser definida como la construcción temporal de compromisos compartidos por actores provenientes de diferentes ambientes estructurales (temporal y relacionalmente), que a través de la interacción del hábito, la imaginación y el juicio reproducen y transforman dichas estructuras, en respuesta interactiva a los problemas planteados por situaciones históricas cambiantes (Emirbayer y Mische, 1998, p. 970), el enfoque crítico de Sommer (2014) apunta a explorar distintas formas de agencia a través de sensibilidades superestructurales, las cuales funcionan como acciones solidarias que usan el performance y el arte para desestabilizar discursos hegemónicos de carácter instrumental. Este es el poder emancipador de una cultura ciudadana que combina arte y pedagogía para retar déficits democráticos y de justicia social, carencias de una modernidad inconclusa que se convierten en agenda política de la esfera pública (Habermas, 2002).

Una manera de maximizar los beneficios de nuestras conexiones en una perspectiva más democrática de vivir el mundo, resulta de responsabilizarse en la doble construcción de una ciudadanía involucrada en su comunidad y una comunidad involucrada con su ciudadanía (Cortina, 1998, p. 92). En esa línea, la creación de documental colaborativo implica el amplio sentido de solidaridad y la generosidad que demuestran los participantes: las comunidades no solo aprenden a crear productos comunicativos de la mano de activistas y creadores profesionales, sino que los papeles se intercambian constantemente; quien en su momento era el pedagogo, toma también el papel de aprendiz de la sabiduría de los miembros de la comunidad, quien en su relato da cuenta de sus anhelos, luchas y deseos. Como dice Jesús Martín Barbero (2008, p. 17): "sólo [sic] existimos en términos de identidad y, por tanto, de diferencia, en la medida en que somos capaces de narrarnos". Esa diferencia es la que nos hace conscientes de que la riqueza cultural está en la diversidad, no en el absolutismo de discursos dominantes.

En ese mismo sentido, la creación de aprendizajes, a través de la interacción comunicativa, desarrolla formas de conocimiento expandido que alimenta la esfera pública para incidir en el empoderamiento ciudadano. No solo se fomenta la creación de capital social -el cual surge de la conformación de redes sociales y normas de reciprocidad y confianza creadas por la comunidad, lo que está íntimamente relacionado con la virtud cívica y se fortalece precisamente cuando se encuentra imbuida en una densa red de relaciones sociales recíprocas (Putnam, 2000)-, sino que la sapiencia práctica y empírica de los participantes desarrolla 
inteligencias colectivas que se pueden replicar en otras intervenciones de sujetos que, sin contar con credenciales institucionales, crean una verdadera rebelión de amateurs que cogestiona el conocimiento de su propia circunstancia (Callon y Rabeharisoa, 2003; Lafuente, Alonso y Rodríguez, 2013).

Así, DOCUPERU rompe con modelos individualistas propios de racionalidades técnicas, para, en su lugar lograr, en la mayoría de los casos, que los participantes en sus intervenciones se conviertan en cooperadores condicionales que se deciden a contribuir en proyectos y acciones colectivas, en tanto observan la participación solidaria de otros, siempre basados en normas de reciprocidad, justicia y confianza (Ostrom, 2000, p. 8). En ese sentido, la tradición cultural participativa latinoamericana presente en las tácticas de intervención de DOCUPERU, al estilo del Teatro Foro de Augusto Boal, empodera a los participantes al devolverles su derecho a la autorrepresentación, vía canales digitales de acceso abierto. Michel de Certeau (2000) decía que las pequeñas victorias de sujetos subordinados sobre grupos dominantes que controlaban la producción de la cultura, se daba a través del consumo, es decir, a través del uso diferenciado y negociado de la misma, algo también observado por Stuart Hall (1973/1980). No obstante, en el entorno de la nueva ecología de medios, también se confeccionan prácticas emancipadoras a través de la propia producción de la cultura, vía lo que Manuel Castells (2009, p. 55) ha denominado como medios masivos de autocomunicación, que desde plataformas digitales permiten la producción y transmisión de contenido de muchos para muchos. Es ahí que prácticas como el documental colaborativo encuentran las rutas desde donde se expresan políticas de representación alternativas que alimentan la resistencia de contrapúblicos subalternos.

\section{Documental colaborativo en tiempos de convergencia mediática}

Al plantear el estudio de los movimientos sociales y de la participación ciudadana a través de los medios, en la era de la revolución digital, interesa cuestionar, en general, estas formas contemporáneas de producción documental y el resurgimiento de las narrativas y metodologías colaborativas, en los tiempos del internet. En lo particular, se quiere someter a discusión y disección un caso que ha hermanado al Perú con México, proyecto que se discutirá al final de este apartado, y que da pie a la integración de la entrevista a José Balado. Para abonar elementos a esta reflexión y discusión, desde lo general, se debe caracterizar primero el giro digital que se enfrenta en los medios, y así mostrar cómo se han generado estos fenómenos, su alcance y utilidad.

Tal vez el primero de los componentes de la convergencia mediática asociada al internet como tecnología que ha generado nuevas narrativas y ha desatado esta noción de la cultura participativa (Jenkins, 2006), sea la democratización de los medios. Esta nueva forma de distribución de contenidos ha sido criticada, e incluso considerada por los enfoques apocalípticos -en términos de Eco- como una visión utópica, por asumir que cualquier persona con una cámara o micrófono y acceso a internet podrá y hará contenidos sin importar la economía política y los intereses de los consorcios comunicativos; y, desde la otra perspectiva, la de los integrados, es justa esta subversión del modelo comunicativo que convierte a estas accesibilidades en un modo viable de comunicación e información.

Estas formas de participación, consumo y producción van adquiriendo carices y formas específicas en los distintos contextos sociales y culturales. "Esta es una cultura que promete nuevas oportunidades de comunicación basada en la inclusión sociocultural más que en la exclusión, ya que trata de redefinir el concepto de creatividad a partir de la participación individual en la coproducción colectiva" (Contreras, 2013, p. 119).

Al tiempo que los productos intentan satisfacer las necesidades de la actualidad, también revitalizan, transforman y comentan su propia tradición mediática. Esta reconfiguración plantea nuevos retos académicos y epistemológicos, lo que nos impele a revisar el pasado teórico y las filiaciones de esta forma del pensamiento latinoamericano, como aconseja Raúl Fuentes (2011). Este esfuerzo que el teórico hace respecto del escenario latinoamericano frente al giro digital puede ser tomado como componente fundamental para pensar el 
cambio del escenario mediático y que, por supuesto, es aplicable al presente objeto de estudio, sobre todo, si se quiere comenzar a caracterizar y matizar los fenómenos en nuestro círculo de acción inmediato.

Ante este cambio en las formas de producción y participación en procesos de creación audiovisual, frente a su sofisticación y diversificación, como apunta Antoni Roig (2010, se deben distinguir los niveles de participación y las formas como cada individuo aporta a estos procesos de producción audiovisual, y precisar y clarificar las características, tanto de este nuevo campo de producción de sentido como de las obras que lo integran. Por ello, es necesario además distinguir el cambio contextual y los retos del giro digital, comprender cómo en la especificidad de la producción documental se hacen visibles estos cambios.

La tecnología al alcance de un porcentaje mayor de la población, para la producción audiovisual y su distribución en plataformas gratuitas en internet, es la parte material que posibilita hablar de la democratización mediática. Dota a los individuos de herramientas para crear contenidos, pero, si se sigue la idea de Fernando Contreras de la coproducción colectiva, darle una cámara, micrófonos y conexión a la red a precios razonables no es el único componente de esta cultura colaborativa.

En ese sentido, como señala Mandy Rose (2014, el concepto de hazlo tú mismo es problemático para el documental. La conciencia de que una aproximación a la producción documental basada en esta postura no está disponible para todo el mundo, nos hace cuestionar sobre el valor de esta noción en el contexto de la compleja producción mediática actual. Desde la óptica de la co-creatividad, hay que defender al documental colaborativo como una respuesta estratégica a la brecha participativa (Jenkins, 2006. Pero hay mucho más en juego en estas prácticas documentales colaborativas. Desde esta perspectiva, el concepto hazlo con otros está mejor equipado para capturar las dinámicas y la relevancia de estos proyectos. Una aproximación hazlo con otros, co-creativa, provee un mecanismo para trabajar el rol histórico del documental en la esfera pública como un espacio abierto de diálogo, así como un escenario para la práctica ciudadana (Rose, 2014.

Es en esta forma específica de producción documental que nos parece útil la reflexión. Y, en ese sentido, también se hace mucho más fácil la búsqueda de las filiaciones y tradiciones de las que estas co-creaciones participan, pues el cine es un producto que, salvo en honrosísimas ocasiones, se hace en grupo. Esta condición de su naturaleza industrial y artística ha luchado enormemente con el otro polo de tensión: la autoría. El hazlo tú mismo empodera al individuo para poder hacer producción solo, pero no le dota de habilidades para cocrear.

$\mathrm{Al}$ considerar cuáles movimientos o producciones son aptas para alinearse como antecedentes de estas formas de producción documental colaborativo en la era digital, no se incluyen todas las formas de cine en la que existan colaboraciones de artistas, a pesar de su relevancia para la industria audiovisual, como por ejemplo, los colectivos fílmicos de las décadas de los veinte y los treinta ${ }^{1}$. Tampoco se trae a colación trabajos colectivos en procesos de taller o experimentación ${ }^{2}$. De alguna u otra forma, todo movimiento cinematográfico es una forma de alineación para la colaboración de distintos autores y productores, desde la fundación de los estudios de Hollywood, pasando por la nueva ola francesa y aterrizando en el Dogma 95.

Tampoco la producción independiente americana o europea, lejos del aparato industrial de los grandes consorcios, interesa como referente de estas prácticas, a pesar de sus grandes aportes al desarrollo narrativo, técnico y organizativo de la producción mediática. Pensando en la historia del cine documental y de los nuevos cines latinoamericanos es que se pueden comenzar a establecer las conexiones y matizar el tipo de colaboración que interesa a este estudio. Serían estos cines surgidos de la precariedad y de la acción social en países como Bolivia, Argentina, Cuba y Brasil que funcionarían como el primer soporte de estas formas de producción que se desea conectar, entre los que aparecen cineastas ineludibles como Jorge Sanjinés, Octavio Getino, Fernando Solanas, Marta Rodríguez, Jorge Silva, Margot Benacerraf, Patricio Guzmán, Raymundo Gleyzer, Glauber Rocha, etcétera. El Grupo Ukamau, el Grupo Cine de la Base o Grupo Cine Labor podrían ser tres ejemplos que se deben citar, entre decenas de ellos.

El compromiso social a través de la crítica a la realidad socioeconómica que viven estos países será uno de los elementos distinguibles de estos proyectos que, además, es pertinente resaltar como uno de los 
sellos característicos. Otro de sus intereses fue la recuperación de las miradas indígenas y la integración de estas culturas, casi invisibilizadas por los discursos nacionalistas de la segunda mitad del siglo XX, en el subcontinente. Este cine de llamado a la acción y de estilo realista es el primer elemento del pasado que se considera puede conectarse directamente con las prácticas documentales desarrolladas por José Balado y el equipo de DOCUPERU.

El otro componente fundamental es el vinculado con las experiencias decolonizadoras en las que se practicó la transferencia de medios para dotar de herramientas y habilidades de producción a comunidades, fundamentalmente indígenas, pero que luego se extendería como práctica habitual de empoderamiento y acción colectiva entre grupos obreros, campesinos y estudiantiles. Este tipo de producción basada en el diálogo entre las comunidades y los cineastas para aproximar la producción a las necesidades comunicativas y expresivas del grupo, barrio o pueblo son el segundo gran soporte y referente para explicar el origen y punto de contacto con la co-creación propuesta por DOCUPERU.

Esta tradición de transferencia sucede en todo el continente, incluso en Norteamérica. En Estados Unidos, inicia con las comunidades Navajo, en 1966 (Singer, 2001) y en Canadá con el proyecto Challenge for Change, que arranca en 1969 (Waugh, Brendan Baker y Winton, 2010), en el resto de países del norte, centro y sur cobrará una gran relevancia. Decenas de proyectos florecerán entre los años ochenta y noventa (Gumucio, 2014), entre los que se pueden destacar Video nas Aldeias (Aufderheide, 1995), las experiencias mexicanas del propio Instituto Nacional Indigenista (Wilson y Stewart, 2008), Ojo de Agua Comunicación y Promedios en Oaxaca y Chiapas, respectivamente (Zamorano y Wammack, 2014), así como el grupo Chaski, en Perú. Gumucio (2014) mapea al menos 55 proyectos de este tipo en América Latina.

Hacia los años noventa, comenzará un recambio generacional y la nueva tecnología en video permitirá dotar de equipos a muchos grupos y comunidades. $\mathrm{Y}$ tras la entrada del internet, estas posibilidades se expandirán y crecerán; darán lugar a una nueva generación de producciones colaborativas que coinciden totalmente con el giro digital. Este nuevo aliento también sucede a pesar de la brecha digital en América Latina (Ardévol, 2014; Córdova, 2011; Flores, 2004; Navarro y Rodríguez, 2014; Suárez, 2009).

Este es el contexto específico en el que surge DOCUPERU, el proyecto de documental colaborativo de José Balado, hace cerca de 15 años. Aunque la base de la propuesta sí es el cine o video de no ficción, muy pronto las necesidades de las comunidades piden ampliar, de manera orgánica, el tipo de productos de comunicación realizados. En el siguiente apartado, se incluye una entrevista con el propio realizador, donde explica este mecanismo de producción participativa de contenidos, así como sus métodos, procesos o guías para hacerlo. Finalmente, comparte algunos resultados del proyecto, entre los que se incluyen dos experiencias de documental colaborativo hechas en Guadalajara (México).

\section{Entrevista con José Balado}

Entrevistador (E): En múltiples ocasiones, has sido claro en mencionar que no existe propiamente una metodología estricta en las intervenciones de DOCUPERU. No obstante, nos gustaría saber si es posible hablar al menos de una definición o una hoja de ruta sobre el tipo de documental colaborativo que realizan en conjunto con las comunidades en donde participan.

José Balado (JB): La organización tiene 14 años y la caravana, unos 10. Comenzamos haciendo video documental porque somos documentalistas y es lo que sabemos hacer. Mi tesis en Brasil la realicé sobre video participativo y ahí fue que vino la idea de implementarlo en Perú. En principio, la caravana surge con la intención pretenciosa de enseñar a la gente a generar contenido de comunicación de forma participativa. No obstante, en el transcurso de todo este tiempo, nos hemos dado cuenta [sic] que no estamos enseñando propiamente contenidos de comunicación, sino que compartimos humanidades y subjetividades, y que a partir de los saberes de los participantes, creamos ahora sí contenidos de comunicación en conjunto: video, 
fotografía, muralismo, fanzine, radio, etcétera. Lo que he aprendido es que no estamos yendo a enseñar video, estamos generando ciudadanía a través de juguetes que es la tecnología. En DOCUPERU, nos reconocemos partícipes del espacio y territorio de las comunidades: no es que solo ellos participen en nuestra experiencia, sino que es desde ambos lados, se es creador y aprendiz de manera indistinta. De hecho, no se invita a participar de lo mío o lo tuyo, pues eso es peligroso porque nos separa: es crear todos juntos. Por tanto, hablar de una metodología es como sonar a manual y lo que hacemos es realmente trabajar en medio de un caos organizado o una organización caótica, es decir, usamos nuestras herramientas en función de las subjetividades con las que nos encontramos y [sic] partir de ahí decidimos cómo trabajar el proyecto. En suma, no tenemos una metodología estricta, sino una plantilla, que a lo mucho nos indica lo que generaremos en cuestiones meramente técnicas. No obstante, lo que haremos con las personas depende de muchas variables que surgen de lo que en DOCUPERU llamamos la Santísima Trinidad, subjetividad, cotidianidad y memoria. Esos son nuestros tres pilares. El primero, subjetividad, es reconocerse como ser único que ve al mundo desde un prisma particular de quien soy, mi formación, la información que me influye, mis determinaciones, etcétera. La cotidianidad es lo que nos rodea, de lo que conozco, mi entorno y mis relaciones con lo habitual, lo cual es un paradigma que tiene posibilidades dramáticas para ser documentado. Ahora bien, cuando se articula la subjetividad y la cotidianidad, entonces llegamos al tercer polo que es la memoria, o más bien dicho, las memorias. Esa es nuestra hoja de ruta, la cual tiene que pasar por esos tres pilares, en donde la gente cuenta su día a día, sus razones para participar en los talleres, su manera de ver el mundo. La gente así se abre con toda naturalidad y nosotros tratamos de expresar esa realidad, bajo su dirección, tal cual ellos la aprecian, cuando se levantan y todo está oscuro, el cantar de los gallos, cuando siembran, etcétera. Lo que hacemos entonces es ponerlo en lenguaje audiovisual en sus propios términos. Incluso, desde la ortodoxia antropológica, nos critican demasiado ya que nos involucramos en el proceso y no nos mantenemos como observadores ajenos, si por ejemplo, un campesino pone una cámara fuera de foco o con mal encuadre, nosotros lo corregimos y le recordamos lo que aprendió en cuestión de técnica audiovisual. Es así que [sic] través de ese diálogo vamos creando contenidos colaborativamente. Esto es, reconocemos mutuamente nuestros saberes y nos ayudamos, ellos nos pueden, por ejemplo, enseñar a sembrar y nosotros a comunicar sus historias.

E: El papel de los mediadores con las comunidades es esencial. Al respecto, ¿cómo se acercan a las comunidades? Al final de cuentas ustedes son agentes externos y requieren de mediadores que los aproximen y que de ahí surja una relación de confianza. De la misma manera, ya que hablas de crear narrativas de manera colaborativa, en donde si bien los participantes tiene la oportunidad de contar historias en sus propios términos, pero apoyados por ustedes como documentalistas, ¿cómo reaccionan dichos participantes a su involucramiento en la producción de la historia?

JB: Hemos aprendido que cuando vamos a intervenir en una comunidad evitamos ir acompañados de ONGs [sic] o de actores gubernamentales porque sabemos que las instituciones son complicadas. Hay que buscar acercarnos, en todo caso, a través de organizaciones de base que existen por toda América Latina, es decir, grupos de gente organizada desde hace mucho tiempo que no dependen del Estado. Como ya tenemos varios años en Perú, por ejemplo, estas organizaciones nos conectan con otras y así sucesivamente. No obstante, puede haber variantes. Por ejemplo, en el caso de Guadalajara, concretamente nuestra participación en el barrio de Santa Cecilia (Santa Chila), nos involucramos a través de una ONG, a la cual investigamos y decidimos que sí podíamos colaborar con ellos, sobre todo porque son conocidos en el barrio y tienen contacto directo con ellos. En este caso, fuimos acompañados de ellos a caminar por el barrio, lo cual nos sirve de termómetro para saber la situación en la que nos encontramos. Ahí, nos topamos con una pandilla que se encontraba fumando marihuana y se acercaron a saludar a nuestro contacto, Aquiles Baeza, el Chino, al cual conocían y era parte del barrio. Lo mismo pasó más adelante con otra pandilla, por lo que eso te da seguridad de que acepten a que entres a su entorno. Es decir, se observa una legitimidad de la persona que introduce al territorio y que nos va a contar otras perspectivas del lugar fuera de los informes institucionales, por lo que es conditio sine qua non para que nosotros entremos, no vamos a lugares si no aseguramos la perspectiva 
desde abajo, del líder comunitario que nos abrió la puerta, la mujer que quiere que vayamos al colegio de su hijo, al profesor que es el héroe del lugar y es el que nos está ayudando porque conoce los liderazgos del sitio, etcétera. Por tanto, para asegurar una intervención efectiva lo primero es conocer y llegar con la gente que legitimará tu entrada al territorio. Después, hablar honesta y directamente sobre quién eres y qué es lo que te interesa saber para que se den cuenta que no eres de la televisión o que deseas hacer solo un reportaje, que no tienes una agenda ni alguien que te paga para realizar un documental, etcétera. Que vean que somos unos locos anarcos que queremos intervenir porque tenemos la disponibilidad y el reto político de hacerlo, no es hacer audiovisual por hacer, es cuestión de transformar a través del mismo. En nuestra experiencia, al externar honestamente nuestras intenciones, la gente se abre y no hemos tenido mayores problemas. A través de esa confianza ganada por sinceridad, las personas te cuentan sus historias y hasta te abren las puertas de sus casas. Con respecto al segundo punto, como realizadores sabemos que lo más bello es el acto de proyección del audiovisual, sentarte a ver, a escuchar, a mirar, a sentir. Que se transmita que no hacemos documentales, sino películas de vida. Por eso uno mira el material y parece aparentemente aburrido porque casi nadie habla sobre películas contemplativas. Sin embargo, lo más lindo es cuando la gente del pueblo se sientan [sic] al momento de la proyección y no están viendo un reportaje, sino la película de su vida. Las personas aparecen como actores que hablan como si fuera un lenguaje de ficción, pero aparece su cocina, donde duerme, donde ocurre lo cotidiano, pero con música, con cortes, con títulos. La gente habla con orgullo de su película, y vive el momento de su representación. Por eso, tenemos que intervenir para que el documental sea bien realizado, depurado, estético, y con ello resulte en el placer de la mirada no solo de los realizadores, sino de la audiencia que los acompaña.

E: Con respecto a la distribución de contenidos, ¿̨por qué han decidido que este material esté disponible manera gratuita por internet? ¿Cómo se les ocurrió que esta sería la plataforma ideal para sus contenidos? Esto es, ¿la distribución del internet les marca un antes y un después?

JB: Ahí está la parte de autorreprentación que nos interesa: visibilizar asuntos que no aparecen en los grandes medios de comunicación. Antes se ponían los nombres de las comunidades en Google y aparecían solo mapas. Ahora aparecen los documentales creados por los propios miembros de la comunidad. Por ejemplo, en el reconocido largometraje peruano Hija de la Laguna, aparece como protagonista Nélida, quien primero participó en un corto que realizamos en 2009 que se llama Yacumama, donde aparece dedicándole una carta hermosa a la madre agua. El corto se conoce bien en Perú, incluso se proyectó ante el presidente y los medios le dieron seguimiento. Ernesto “Tito" Cabellos, el director de Hija de la Laguna, ve el corto y conoce a Nélida. Lo demás es historia. Así pues, lo que hacemos a través de internet es visibilizar los temas que están en la agenda pública, y si no están, ponerlos. Hace dos años trabajamos en el tema de esterilización forzadas en la época de Alberto Fujimori en Perú; 300.000 mujeres fueron esterilizadas y a 50.000 hombres les practicaron la vasectomía, todo hecho de manera ilegal en clara violación de derechos humanos. Como eran campesinos indígenas iletrados y pobres, parecía no interesarle a nadie. Si hubiesen sido dos mujeres de Lima, esto hubiera sido un escándalo, pero ante sus condiciones de exclusión social no hubo la más mínima cobertura mediática. Ante este doloroso agravio, nos dimos a la tarea de generar contenido, hicimos videos y fotos para posteriormente hacer giras en centros culturales y universidades. Se lo presentamos a organizaciones feministas, de derechos humanos, entre otros, para visibilizar este asunto. De la misma manera, lo empezamos a distribuir a través de plataformas digitales por internet, y no solo eso, enviamos copias a muchas partes para tener acceso a circuitos y foros alternativos. Y todo esto de manera gratuita, pues lo que hacemos en DOCUPERU es de libre acceso, hackeable, pirateable, porque nuestra idea es salir de lógicas individualistas de mercado, lo nuestro es free domain y free use.

E: En ese sentido y por más obvia que resulte la respuesta, ¿̨podemos entonces considerar al documental colaborativo como un acto político? 
JB: Definitivamente. Creo que debemos repensar qué es la política y lo público. Es cambiar el paradigma, no solo pensar en términos de partido y la participación a través de ellos, sino politizar al cuerpo, la voz, las expresiones sociales. Sí, el documental colaborativo es una forma de empoderamiento político.

E: La pregunta viene al caso sobre todo porque en Latinoamérica tenemos toda una tradición de luchas que parten desde lo local, desde abajo, desde las comunidades. Son los sitios donde surgen estrategias emancipadoras para romper con discursos dominantes y democratizar la vida social in toto.

JB: Absolutamente. Es por eso que para nosotros cobra tanta importancia el término de territorio, no solo el geográfico, sino el de la vida, el cuerpo, las relaciones sociales. Por ejemplo, las mujeres que fueron esterilizadas sufrieron una invasión del territorio de su cuerpo y ni qué decir del alma. Y esta demarcación territorial es expuesta a través del audiovisual como historias de vida. Una metáfora que me gusta usar es que en DOCUPERU no hacemos esto para jugar en los grandes estadios, sino que nos interesan las pichangas (futbol de barrio, las que se dan debajo de los puentes, en los parques, los barrios; lo que queremos es que el audiovisual sea como un montón de pichangas que exista en todos lados, y no solo aquel que va a los grandes festivales, o hecho para la televisión. Lo nuestro entonces es hacer del documental una política de transgresión.

E: $\mathrm{Al}$ respecto, si hay tanto énfasis en el territorio, ¿acaso el internet no desterritorializa esta práctica de documental participativo? ¿Cómo subvertir esa parte abstracta de no conocer el territorio, de que todo da igual porque es un sitio en otro país conectado por un servidor, y que incluso se comparte de manera global?

JB: Más bien son nuevas formas de territorialidad que nos conecta con diversos sitios, pero con intereses comunes. Por ejemplo, hemos creado toda una red de profesionales de distintas disciplinas con las que interactuamos y que les interesa crear vínculos virtuales con nosotros. Es decir, pueden existir prácticas que despersonalizan, pero al final lo importante es cómo nos conectarnos. La tecnología ha redefinido esas posibilidades de conexión, pero otra vez, dichas tecnologías de información son juguetes que no actúan por voluntad propia, es nuestra responsabilidad jugar juntos. Por eso, nuestra insistencia en que les llamemos así, juguetes, el acceso a la tecnología puede resultar en nuevas formas de separación social, por lo que es muy importante que usemos esa terminología porque todos tenemos juguetes. El reto, en todo caso, es saber cómo lograr interactuar con tus juguetes y los míos. De acuerdo a lo anterior y en el mismo sentido de usar el lenguaje de otra manera para mantenernos en una relación horizontal y sin jerarquías, esto nos sirve para acercamos a la gente de las comunidades en una relación de iguales. Como estamos jugando, todo el tiempo produciendo contenidos colaborativos, nadie está sobre de nadie, estamos compartiendo saberes. Cuando alguien me mira y me dice: “ioiga profesor!”, inmediatamente le contesto que aquí no soy un profesor, que me hable por mi nombre para que con mayor confianza me hable de su vida. Esto es, no interactuamos nunca a partir de relaciones de poder porque ello va en contra de la transmisión de conocimiento; por el contrario, hablamos como cotidianamente lo hacen, de manera coloquial, sin falsas posturas, con mala palabras incluso. Es decir, cualquier 'buevón' puede construir conocimiento. Esto lo hacemos también para que la próxima vez que venga una ONG o autoridad con ellos y no se hablen como iguales, ellos también cuestionen dichas formas de poder a través la enseñanza. No son bobos, ya han sido engañados muchas veces y las organizaciones de base saben quién les habla desde la confianza y quién desde el privilegio. Lo que hacemos al final es estar jugando todo el tiempo, mirándonos de frente, tocándonos, nos queda claro que para humanizar el producto de comunicación, hay que humanizar el proceso de la comunicación.

E: Una vez que termina la intervención en las comunidades y se regresa tiempo después, ¿qué sucede en el reencuentro? ¿Qué observas diferente en el trato de pasar de un desconocido a alguien con quien compartieron su vida? ¿Cómo valorar el capital social construido?

JB: Cuando regresamos, ya sea porque volvemos a una segunda intervención, o porque pasamos de vacaciones, o porque voy directamente a ese lugar a visitarlo con mi hijo, los vínculos humanos permanecen de manera increíble. Sigo siempre en contacto con ellos, nos llamamos, nos escribimos. Se convierten en mis amigos y amigas y, dependiendo del proyecto, existen posibilidades de seguir interviniendo en otros niveles, más profundo. Por eso es que hemos creado varios proyectos participativos. Tenemos la Caravana documental, 
que es la ruta itinerante de un mes, donde viajamos y el objetivo es visibilizar temáticas y experiencias de gente, y poder tener sus contenidos disponibles en internet, los cuales pueden ser variables y surgen a partir de la interacción. Otro tipo de proyecto que realizamos se llama Medios que conmueven. Es participativo también, pero se realiza donde hay una temática puntual que no está visible en el país, y que nadie la está cubriendo. Eso fue lo que hicimos con el caso de las esterilizaciones forzadas en Perú en donde estuvimos trabajando durante dos años, y nadie de DOCUPERU cobró un solo centavo. Era un trabajo que teníamos y queríamos hacer, y más aún que más de la mitad de los miembros de DOCUPERU son mujeres. La discusión de género está siempre presente, y las compañeras decidieron que había que hacer algo en Huancabamba. Así pues, lo asumimos y nos fuimos todo ese tiempo a trabajar con mujeres en diferentes niveles, para generar opinión pública en el tema. A diferencia pues de la Caravana itinerante, esto fue una cuestión política objetiva. De la misma manera, se realizó el proyecto de Cajamarca por tres años sobre medio ambiente y recursos acuíferos en la zona, estuvimos ahí incluso antes de que se anunciara el proyecto minero Conga que contaminaría terriblemente toda la zona. Hablamos con campesinos y granjeros y les enseñamos a usar cámaras y diseñar blogs para que dieran a conocer su lucha ecológica.

El tercer tipo de proyecto que realizamos, a partir de 2014, se llama Mochila documental. En este se interviene en sitios no considerados en una Caravana documental que tiene ya especificada una ruta concreta con comunidades específicas, ni presentan problemáticas puntuales como en el caso de Medios que conmueven. Son espacios que intervenimos y que no estaban propiamente en nuestra agenda, pero en los que consideramos se puede colaborar. Al respecto, hemos trabajado en Cuzco en los últimos dos años en la creación de una escuela itinerante de formación de líderes en creación audiovisual. Cooperación Belga nos ha pagado dos años seguidos para traer unas 20 personas de diferentes partes del territorio nacional, que son hombres, mujeres, adolescentes, gente mayor, quienes son líderes en sus comunidades y tienen una formación política increíble, pero no conocen el manejo de audiovisuales y medios de comunicación. Así, los capacitamos unas semanas para entrenarlos en la creación de documental, stop motion, blogs, diseño de campañas de medios, estrategias de medios, es dotar a estos líderes en el uso de los mismos. Así pues, creamos Mochila documental, porque nosotros ponemos nuestra mochila y nos vamos a donde la gente nos necesita para enseñar el uso de medios.

E: Dentro de este panorama de los tres proyectos ¿qué es lo que se ha hecho concretamente en Guadalajara?

JB: En Guadalajara, hacemos Mochila documental porque surge de la idea de ir a otros lados a transferir experiencias y generar productos, con la idea de crear nodos de DOCUPERU en distintos sitios, las pichangas de las que hablaba.

E: ¿Has encontrado algunas diferencias de las experiencias realizadas en Perú con respecto a las de Guadalajara?

JB: Cuando hablamos de subjetividades y cotidianidades, hay tantas cosas iguales que van más allá de la geografía. Es como los cinéfilos, ¿de qué hablan al final las grandes películas de la historia?, de los mismos temas. Aunque Kurozawa hablaba solo de Japón, sus películas las pueden ver un iraní, un colombiano, o de donde sea, y se va a conectar porque están hablando de la condición humana. Fellini, más italiano no podía ser, nos comunica sin problema. Es decir, nos damos cuenta también [sic] que mientras más te apegas a lo básico, a la condición humana, más te conectas con los demás. Participé, hace años, en una gira para la cual me contrató la OEA para dar talleres en Centro América a expandilleros que estaban trabajando ahora en organizaciones sociales. Fui a Panamá, Nicaragua, El Salvador, Honduras y Guatemala. Les pasaba documentales de Cajamarca, sobre campesinas buscando obtener un poco de leche para sus hijos. Aún con todas las condiciones particulares de la comunidad de la selva peruana, se generaron grandes discusiones en las que estos chicos expandilleros se reflejaban. Así, uno se da cuenta [sic] que son los mismos temas, el quechua y los sobreros son ajenos a estos chicos urbanos, pero son los mismos temas de explotación que a todos nos afectan. Por tanto, lo mismo sucede entre México y Perú, que no solo nos une el castellano o provenir de culturas ancestrales, sino toda una serie de problemáticas políticas complejas sobre corrupción 
y explotación. Al final, estamos hablando de transgresiones más allá de geografías, pues los territorios se conectan. Tenemos otras prácticas que hemos estado implementando con la gente en cada día de intervención, antes de empezar el día, así como después del almuerzo, hacemos dinámicas grupales que tocan el cuerpo, hablan de territorio, hacemos mapas, manualidades, etcétera, para juntar a la gente con temas que no son propiamente del documental, pero que nos conectan subjetivamente de otra manera. Así pues, de verdad, no sentí ninguna diferencia acá en Guadalajara. Le comenté a los colegas mexicanos que me acompañaban, que al estar en Santa Chila me sentía como si estuviera en territorio peruano, no me era ajeno porque nos conectábamos tanto con el equipo como con el barrio, como si fuésemos una extensión más del espacio. Hicimos ocho documentales durante una semana, estuvimos como locos, y la verdad es que es más lo que nos une que las diferencias que nos separan.

\section{Conclusiones}

Es a la conquista de estos territorios reales y figurados, mencionados por Balado, que se pueden lanzar proyectos co-creativos como DOCUPERU. Formas artísticas y productos mediáticos que derivan en agencia y participación ciudadana, en nuevas formas de conexión a través del internet y de recorrer la geografía latinoamericana. Ahí donde las metodologías deben ceder paso al diálogo entre sujetos y a la adecuación de los contenidos y las experiencias a las necesidades de la comunidad.

Esta dinámica de autorrepresentación, de exploración audiovisual de la vida cotidiana y la memoria son formas potentes de legado cultural, de empoderamiento de los individuos y de fortalecimiento de las democracias en el subcontinente. El acceso a nuevos medios y espacios de difusión deben ir acompañados de una sólida red de colaboración, de ayuda y de solidaridad mutua. Esto, nos parece, es lo que sucede en las experiencias colaborativas derivadas de las intervenciones de DOCUPERU.

\section{Referencias}

Ardévol, E. (2014. Cine y video indígena ¿̨hacia una comunicación alternativa? En E. Ardévol y N. Muntañola (coords.), Representación y cultura audiovisual en la sociedad contemporánea (pp. 316-338). Barcelona: Universitat Oberta de Catalunya.

Aufderheide, P. (1995). The video in the Villages Project: Video making with and by Brazilian Indians. Visual Anthropology Review, 11(2), 83-93. https://doi.org/10.1525/var.1995.11.2.83

Benhabib, S. (2002). The claims of culture: Equality and diversity in the global era. Princeton: Princeton University Press.

Boggs, C. (1986). Social movements and political power. Philadelphia, PA: Temple University Press.

Callon, M., y Rabeharisoa, V. (2003). Research 'in the wild' and the shaping of new social identities. Technology in Society, 25(2), 193-204. https://doi.org/10.1016/S0160-791X(03)00021-6

Castells, M. (2009). Communication power. Oxford: Oxford University Press.

Certeau, M. de (2000). La invención de lo cotidiano. México: Universidad Iberoamericana/Instituto Tecnológico y de Estudios Superiores de Occidente.

Chambers, S. (2003). Deliberative democratic theory. Annual Review of Political Science, 6, 307-326. https://doi.org /10.1146/annurev.polisci.6.121901.085538

Contreras, F. (2013). La colaboración en la esfera pública digital. En F. Sierra (ed.), Ciudadanía, tecnología y cultura (pp. 119-149). Barcelona: Gedisa.

Córdova, A. (2011). Estéticas enraizadas: aproximaciones al video indígena en América Latina. Comunicacióny Medios, 24, 81-107. https://doi.org/10.5354/0719-1529.2012.19895

Cortina, A. (1998). Ciudadanos del mundo. Hacia una teoría de la ciudadania. Madrid: Alianza. 
Dryzek, J. (2009). Democratization as deliberative capacity building. Comparative Political Studies, 42(11), 1379-1402. https://doi.org/10.1177/0010414009332129

Elster, J. (comp.). (2001). La democracia deliberativa. Barcelona: Gedisa.

Emirbayer, M., y Mische, A. (1998). What is agency? The American Journal of Sociology, 103(4), 962-1023. https:// doi.org/10.1086/231294

Flores, C. Y. (2004). Indigenous video, development and shared anthropology: A collaborative experience with Maya Q'eqchi' filmmakers in postwar Guatemala. Visual Anthropology Review, 20(1), 31-44. https://doi.org/10.1525 /var.2004.20.1.31

Fraser, N. (1992). Rethinking the public sphere: A contribution to the critique of actually existing democracy. En C. Calhoun (ed.), Habermas and the public sphere (pp. 109-142). Cambridge, MA: Massachusetts Institute of Technology Press.

Fuentes, R. (2011). Pensamiento comunicacional latinoamericano y convergencia digital. Retos epistemológicos y académicos. En C. del Valle, F. J. Moreno y F. Sierra (eds.), Cultura latina y revolución digital (pp. 41-68). Barcelona: Gedisa.

Fung, A. (2005). Deliberation before the revolution. Toward an ethics of deliberative democracy in an unjust world. Political Theory, 33(2), 397-419. https://doi.org/10.1177/0090591704271990

Gumucio, A. (2014). El cine comunitario en América Latina y el Caribe. Bogotá: Fundación del Nuevo Cine Latinoamericano.

Habermas, J. (1987). The theory of communicative action. Boston, MA: Beacon Press.

Habermas, J. (1989). The structural transformation of the public sphere: An inquiry into a category of bourgeois society. Cambridge, MA: Massachusetts Institute of Technology Press.

Habermas, J. (2002). Modernity: An incomplete project. En H. Foster (ed.), The anti-aesthetic. Essays on postmodern culture (pp. 3-15). Washington, DC: Bay Press.

Hall, S. (1980). Encoding/decoding [(1973). Encoding and Decoding in the Media Discourse (Stencilled Occasional Paper, no.7). Birmingham: Centre for Contemporary Cultural Studies, University of Birmingham]. En S. Hall, D. Hobson, A. Lowe y P. Willis (eds.), Culture, media, language: Working papers in cultural studies, 1972-79 (pp. 128-138). Londres: Hutchinson \& Company Publishers.

Held, D. (2007). Modelos de democracia. Madrid: Alianza.

Jenkins, H. (2006). Convergence culture. Nueva York: New York University Press.

Lafuente, A., Alonso, A., y Rodríguez, J. (2013). ;Todos sabios! Madrid: Cátedra.

Martín Barbero, J. (2008). Diversidad cultural y convergencia digital. IC. Revista Cientifica de Información y Comunicación, 5, 12-25. Recuperado de http://www.icjournal-ojs.org/index.php/IC-Journal/article/view/265 $/ 261$

Melucci, A. (1996). Challenging codes: Collective action in the information age. Cambridge, MA: Cambridge University Press.

Navarro, V., y Rodríguez, J. C. (eds.). (2014). New documentaries in Latin America. Nueva York: Palgrave McMillan.

Offe, C. (1984). Contradictions of the Welfare State. Cambridge, MA: Massachusetts Institute of Technology Press.

Ostrom, E. (2000). Crowding out citizenship. Scandinavian Political Studies, 23(1), 3-16. https://doi.org/10.1111/ $1467-9477.00028$

Putnam, R. (2000). Bowling alone. The collapse and revival of American community [version Kindle]. Nueva York: Simon \& Schuster, Inc.

Roig, A. (2010). La participación como bien de consumo: una aproximación conceptual a las formas de implicación de los usuarios en proyectos audiovisuales colaborativos. Anàlisi: Quaderns de Comunicació i Cultura, 40, 101-114. https://doi.org/10.7238/a.v0i40.1147

Rose, M. (2014). Making publics: Documentary as do-it-with-others citizenship. En M. Boler y M. Ratto (eds.), DIY citizenship: Critical making and social media (pp. 201-212). Boston: Massachusetts Institute of Technology Press. 
Singer, B. R. (2001). Wiping the war paint off the lens. Native American film and video [version Kindle]. Minneapolis: Minnesota University Press.

Sommer, D. (2014). The work of art in the world. Civic agency and public humanities. Durham, NC: Duke University Press.

Sommer, D. (ed.). (2006). Cultural agency in the Americas. Durham, NC: Duke University Press.

Suárez, B. (2009). El documental social como proyecto de desarrollo frente a los intereses de las industrias culturales en la era digital. Signo y Pensamiento, 28(54), 208-227. Recuperado de https://revistas.javeriana.edu.co/index. $\mathrm{php} /$ signoypensamiento/article/view/4535

Velasco, J. C. (2006). Deliberación y calidad de la democracia. Claves de razón práctica, 167, 36-43. Recuperado de ht tp://www.revistasculturales.com/revistas/15/claves-de-razon-practica/num/167/

Waugh, T., Brendan Baker, M., y Winton, E. (eds.). (2010). Challenge for Change. Activist Documentary at the National Film Board of Canada [version Kindle]. Montreal: McGill-Queen's University Press.

Wilson, P., y Stewart, M. (2008). Global indigenous media. Cultures, poetics, and politics. Durham, NC: Duke University Press.

Zamorano, G., y Wammack, B. (2014). El audiovisual indígena en México y sus aportes al género documental. En A. Muñoz y C. Curiel (eds.), Reflexiones sobre el cine mexicano contemporáneo documental (Vol. 2, pp. 129-147). México: Cineteca Nacional.

\section{Notas}

* Artículo de investigación científica Esta investigación parte de la colaboración de los autores con el equipo de DOCUPERU para la impartición de talleres de documental colaborativo en la Zona Metropolitana de Guadalajara, México.

1 Pensamos en el tren cinematográfico de Aleksandr Medvedkin o el propio grupo de los tres de Dziga Vertov o las misiones pedagógicas en España con José Val del Omar y García Lorca.

2 Nos viene a la mente las filmaciones en el estudio de arte de Warhol, La Fábrica, por ejemplo.

\section{Licencia Creative Commons CC BY 4.0}

Cómo citar este artículo: Leetoy, S., y Zavala Scherer, D. (2019). Subjetividad, cotidianidad y memoria: la propuesta de documental colaborativo de José Balado. Signo y Pensamiento, 38(74). https://doi.org/10.111 44/Javeriana.syp38-74.scmp 